University of Nebraska - Lincoln

DigitalCommons@University of Nebraska - Lincoln

\title{
MPK6, Sphinganine and the LCB2a Gene from Serine Palmitoyltransferase are Required in the Signaling Pathway That Mediates Cell Death Induced by Long Chain Bases in Arabidopsis
}

\author{
Mariana Saucedo-García \\ Universidad Nacional Autónoma de México \\ Arturo Guevara-Garcia \\ Universidad Nacional Autónoma de México \\ Ariadna González-Solís \\ Universidad Nacional Autónoma de México \\ Felipe Cruz-García \\ Universidad Nacional Autónoma de México \\ Sonia Vázquez-Santana \\ Universidad Nacional Autónoma de México \\ Follow this and additional works at: hetps://digitalcommons.unl.edu/plantscifacpub \\ Part of the Plant Biology Commons, Plant Breeding and Genetics Commons, and the Plant Pathology \\ Eefripthpage for additional authors
}

Saucedo-García, Mariana; Guevara-Garcia, Arturo; González-Solís, Ariadna; Cruz-García, Felipe; VázquezSantana, Sonia; Markham, Jennifer E.; Lozano-Rosas, M. Guadalupe; Dietrich, Charles R.; Ramos-Vega, Maricela; Cahoon, Edgar B.; and Gavilanes-Ruíz, Marina, "MPK6, Sphinganine and the LCB2a Gene from Serine Palmitoyltransferase are Required in the Signaling Pathway That Mediates Cell Death Induced by Long Chain Bases in Arabidopsis" (2011). Faculty Publications from the Center for Plant Science Innovation. 199.

https://digitalcommons.unl.edu/plantscifacpub/199

This Article is brought to you for free and open access by the Plant Science Innovation, Center for at DigitalCommons@University of Nebraska - Lincoln. It has been accepted for inclusion in Faculty Publications from the Center for Plant Science Innovation by an authorized administrator of DigitalCommons@University of Nebraska - Lincoln. 


\section{Authors}

Mariana Saucedo-García, Arturo Guevara-Garcia, Ariadna González-Solís, Felipe Cruz-García, Sonia Vázquez-Santana, Jennifer E. Markham, M. Guadalupe Lozano-Rosas, Charles R. Dietrich, Maricela Ramos-Vega, Edgar B. Cahoon, and Marina Gavilanes-Ruíz 


\section{MPK6, sphinganine and the $\angle C B 2 a$ gene from serine palmitoyltransferase are required in the signaling pathway that mediates cell death induced by long chain bases in Arabidopsis}

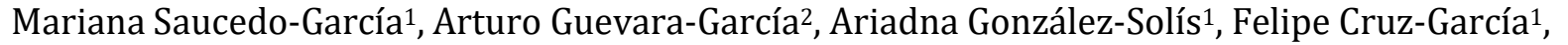
Sonia Vázquez-Santana ${ }^{3}$, Jennifer E. Markham ${ }^{4}$, M. Guadalupe Lozano-Rosas ${ }^{1}$, Charles R. Dietrich, Maricela Ramos-Vega ${ }^{2}$, Edgar B. Cahoon ${ }^{5}$, and Marina Gavilanes-Ruíz ${ }^{1}$

${ }^{1}$ Dpto. de Bioquímica, Fac. de Química, Universidad Nacional Autónoma de México, México D.F., 04510 México; ${ }^{2}$ Dpto. de Biología Molecular de Plantas, Inst. de Biotecnología, Universidad Nacional Autónoma de México, Cuernavaca 62271, Morelos, México; ${ }^{3}$ Dpto. de Biología Comparada, Fac. de Ciencias, Universidad Nacional Autónoma de México, México D.F., 04510 México; ${ }^{4}$ Donald Danforth Plant Science Center, St Louis, Missouri 63132, USA; ${ }^{5}$ Center for Plant Science Innovation and Department of Biochemistry, University of Nebraska-Lincoln, E318 Beadle Center, 1901 Vine St., Lincoln, Nebraska 68588, USA

\footnotetext{
Summary

- Long chain bases (LCBs) are sphingolipid intermediates acting as second messengers in programmed cell death (PCD) in plants. Most of the molecular and cellular features of this signaling function remain unknown.

- We induced PCD conditions in Arabidopsis thaliana seedlings and analyzed LCB accumulation kinetics, cell ultrastructure and phenotypes in serine palmitoyltransferase (spt), mitogen-activated protein kinase (mpk), mitogen-activated protein phosphatase (mkp1) and lcb-hydroxylase (sbh) mutants.

- The Icb2a-1 mutant was unable to mount an effective PCD in response to fumonisin B1 (FB1), revealing that the $L C B 2 a$ gene is essential for the induction of PCD. The accumulation kinetics of LCBs in wild-type (WT) and Icb2a-1 plants and reconstitution experiments with sphinganine indicated that this LCB was primarily responsible for PCD elicitation. The resistance of the null mpk6 mutant to manifest PCD on FB1 and sphinganine addition and the failure to show resistance on pathogen infection and MPK6 activation by FB1 and LCBs indicated that MPK6 mediates PCD downstream of LCBS.

- This work describes MPK6 as a novel transducer in the pathway leading to LCB-induced PCD in Arabidopsis, and reveals that sphinganine and the $\angle C B 2 a$ gene are required in a PCD process that operates as one of the more effective strategies used as defense against pathogens in plants.
}

Author for correspondence:

Marina Gavilanes-Ruíz

Tel: +5256225376

Email: gavilan@unam.mx

Received: February 7, 2011

Accepted: March 14, 2011

New Phytologist (2011) 191: 943-957

doi: 10.1111/j.1469-8137.2011.03727.x

\section{Introduction}

Programmed cell death (PCD) is a necessary and recurrent process during the plant life cycle, participating in differentiation, immunity and senescence (Greenberg, 1996; Turner et al., 2007; Mur et al., 2010; Peer et al., 2010). Recently, sphingolipids have been revealed as PCD mediators in plants (Shi et al., 2007; Wang et al., 2008; Pata et al., 2009), as known previously in yeast and mammalian cells (Liu K et al., 2005; Hannun \& Obeid, 2008). Given
Key words: fumonisin B1, long chain bases, MAPK signaling, MPK6, programmed cell death, serine palmitoyltransferase, sphingoid bases, sphingolipids. the vast and complex repertoire of sphingoid compounds revealed by new analytical tools (Markham et al., 2006; Markham \& Jaworski, 2007) and by the existence of a plethora of enzymes that catalyze sphingolipid metabolism (Supporting Information Fig. S1) (Chen et al., 2009), the assignment of precise signaling roles to specific sphingoid species and a knowledge of their mechanisms of action in PCD and in other cell functions are particularly challenging in plants. 
As signaling molecules, phosphorylated sphingoid bases, or long chain bases (LCBs-P), regulate stomatal closure ( $\mathrm{Ng}$ et al., 2001; Coursol et al., 2003, 2005; Townley et al., 2005; Worrall et al., 2008), whereas long chain bases (LCBs) and ceramides regulate PCD (Shi et al., 2007; Wang et al., 2008; Lachaud et al., 2010). In this regard, evidence of the involvement of sphingoid compounds has been known for some time. For example, fumonisin B1 (FB1) is a mycotoxin that disrupts sphingolipid biosynthesis (Fig. S1) and elicits PCD (Stone et al., 2000; Merrill et al., 2001). In Arabidopsis, FB1-induced PCD is triggered by increased LCB levels, as demonstrated by a mutation in the LCB1 gene (Shi et al., 2007) that encodes one of the two subunits of serine palmitoyltransferase (SPT), a key enzyme in the de novo synthesis of sphingolipids (Gable et al., 2002) (Fig. S1). Sphingolipids in plants also participate in the PCD of defense against pathogens, called the hypersensitive response (HR-PCD), as shown by mutants exhibiting a premature cell death phenotype and other defense responses (Brodersen et al., 2002; Liang et al., 2003). However, despite all the work carried out on this issue, PCD elicited by LCBs is largely unknown in terms of the molecular components of the pathway and of the changes to the cell during this process.

We hypothesized that LCBs might induce mitogen-activated protein kinase (MAPK) cascades leading to $\mathrm{PCD}$ induced by FB1, based on the following evidence: (1) FB1 increases MPK activity in animal cells (Wattenberg et al., 1996; Pinelli et al., 1999); (2) infection or treatment with elicitors leading to HR-PCD induces the activation of salicylic acid (SA)-induced (SIPK) and wound-induced (WIPK) protein kinases in Nicotiana tabacum (Zhang et al., 1998, 2000) and of MPK6 and MPK3 (SIPK and WIPK Arabidopsis orthologs, respectively) (Desikan et al., 2001; Ren et al., 2008); (3) Arabidopsis plants overexpressing MEK4 and MEK5 (that are MAPKK) show an HR-PCDlike phenotype and a prolonged activation of the MPK3 and MPK6 kinases (MEK4 and MEK5 downstream targets) (Ren et al., 2002); and (4) the rice MPK6 (OsMPK6) ortholog of AtMPK6 is activated by a sphingolipid elicitor from a blast fungus (Lieberherr et al., 2005).

In this study, we induced PCD conditions in Arabidopsis seedlings using FB1 in several genetic backgrounds in order to investigate how LCB accumulation kinetics are associated with molecular and cell death features. Our results reveal that the $L C B 2 a$ gene, the dihydroxylated LCB sphinganine (d18:0) and MPK6 are important contributors to the LCBinduced $\mathrm{PCD}$, which can be physiologically manifested as an HR-PCD. We also found that LCB- and MPK6induced PCD involves extensive disorganization of endomembranes and features of autophagic cell death. MPK6 is the first kinase described as a transducer in the LCBmediated PCD in plants.

\section{Materials and Methods}

\section{Plant growth conditions and treatments}

For the germination and growth of Arabidopsis thaliana (L.) plants, 3-wk-old Arabidopsis seedlings were transferred to Gamborg plates supplemented with FB1 (Sigma-Aldrich Corp., St Louis, MO, USA) and incubated at $22^{\circ} \mathrm{C}$ under constant light $\left(11.5 \mu \mathrm{mol} \mathrm{m}^{-2} \mathrm{~s}^{-1}\right)$ for the indicated concentrations and times. For myriocin (Sigma-Aldrich Corp.) treatment, seedlings were transferred to solid medium supplemented with $100 \mathrm{nM}$ myriocin and exposed during $5 \mathrm{~d}$ before FB1 treatment. The details are described in Methods S1.

\section{Isolation of T-DNA insertion lines and construction of mutant lines}

The previously characterized T-DNA insertional mutants lcb2a-1 (SALK_061472), lcb2a-2 (GABI 216-D07), lcb2a-3 (SAIL_706-A05) (Dietrich et al., 2008) and sbh1-1 (SALK_090881) (Chen et al., 2008) were obtained from the Arabidopsis Biological Resource Center (Ohio State University, Columbus, OH, USA). RNAi-silenced $m p k 3$ plants were generated by transgenesis as described in Methods S1. An mpk6-2 homozygous line was recovered by self-crossing heterozygous seedlings from the SALK 073907 insertional mutant collection stock (see details in Methods S1). The $m k p 1$ single mutant and $m p k 3 m k p 1$ and $m p k 6 m k p 1$ double mutants were kindly donated by Marina A. González Besteiro from the Roman Ulm Laboratory (University of Geneva, Switzerland) (Ulm et al., 2001). The presence of T-DNA insertions was verified as described in Methods S1.

\section{Detection of nuclear DNA fragmentation}

DNA from controls and seedlings treated with FB1 for $5 \mathrm{~d}$ was stained with 4',6-diamidino-2-phenylindole (DAPI; Sigma-Aldrich Corp.) and the free $3^{\prime}-\mathrm{OH}$ groups in the DNA were subjected to terminal deoxynucleotidyl transferase-mediated UTP nick end labeling (TUNEL) reaction, as described in Methods S1.

\section{Ultrastructural analysis}

FB1-treated and untreated seedlings were fixed, dehydrated, embedded, sectioned and observed as described in Methods S1.

\section{LCB analysis}

Seedlings were collected after treatment with $10 \mu \mathrm{M}$ FB1 for $0,1,4,8,12$ and $72 \mathrm{~h}$, frozen in liquid nitrogen and 
later lyophilized. Quintuplicates of these treatments were independently performed and processed. LCBs-P were extracted from $30 \mathrm{mg}$ of the lyophilized tissue (dry weight), followed by separation, identification and quantification through reverse-phase high-performance liquid chromatography coupled with electrospray ionization tandem mass spectrometry (HPLC ESI-MS/MS), as described in Markham \& Jaworski (2007).

\section{Protein extraction, kinase assays and immunoblot analyses}

Soluble protein extracts were obtained from seedlings and subjected to in-gel kinase activity assays, and immunoblots were performed as described in Methods S1.

\section{Treatment with exogenous LCBs}

Three-wk-old Arabidopsis wild-type (WT) or mutant seedlings grown in Petri dishes $(3.5 \mathrm{~cm}$ in diameter) were sprayed with $5 \mu \mathrm{M}$ d18:0 (Sigma-Aldrich Corp.), $5 \mu \mathrm{M}$ t18:0 (Sigma-Aldrich Corp.) or $5 \mu \mathrm{M}$ d18:0-P (Avanti Polar Lipids, Inc., Alabaster, AL, USA) solution ( $0.7 \mathrm{ml}$ per dish). All compounds were dissolved in $0.04 \%$ (v/v) Silwet L-77 (Chemtura Corporation México, S. de RL de C.V. México, D.F., México). Controls sprayed with Silwet solution were included. Seedlings were kept under continuous light $\left(11.5 \mu \mathrm{mol} \mathrm{m}^{-2} \mathrm{~s}^{-1}\right)$ at $22^{\circ} \mathrm{C}$ and phenotypic changes were recorded photographically.

\section{Bacterial inoculation and in planta growth determi- nation}

Fresh cultures of Pseudomonas syringae pv. tomato avrRpm1 were obtained and resuspended in $10 \mathrm{mM} \mathrm{MgCl} 2$. The suspension $\left[10^{7}\right.$ colony-forming units $\left.(\mathrm{cfu}) \mathrm{ml}^{-1}\right]$ was sprayed over the Petri dishes $(2.0 \mathrm{ml}$ per 8 -cm-diameter dish) containing the Arabidopsis WT, lcb2a-1 or mpk6 mutant seedlings, which had been exposed, or not, to $10 \mu \mathrm{M}$ FB1 for $12 \mathrm{~h}$ (under continuous light, $11.5 \mu \mathrm{mol} \mathrm{m}^{-2} \mathrm{~s}^{-1}$ ). Seedling samples were collected at the indicated times and ground in $10 \mathrm{mM} \mathrm{MgCl} 2$; serial dilutions were plated in Kings B medium supplemented with $40 \mu \mathrm{g} \mathrm{ml}^{-1}$ rifampicin and $50 \mu \mathrm{g} \mathrm{ml}^{-1}$ kanamycin, incubated at $29^{\circ} \mathrm{C}$ and the number of colonies was counted after $48 \mathrm{~h}$ (Yang et al., 2002).

\section{Results}

Two approaches used to generate low or high accumulation of endogenous LCBs

To gain an insight into the pathway between LCB accumulation and PCD, we used two systems that allowed an increase or decrease in endogenous LCBs in Arabidopsis seedlings. In one system, the pharmacological approach, seedlings were exposed to FB1 to generate an accumulation of LCBs that eventually produced tissue cell death (Fig. S1). Moreover, myriocin, an SPT inhibitor, was used to decrease LCB synthesis and to prevent LCB accumulation (Fig. S1). FB1 treatment caused generalized cell death in the WT seedling tissues, which was prevented by pretreatment with myriocin (Fig. 1a), suggesting that FB1 decreased the viability of WT seedlings through the production of high LCB levels.

Another strategy to decrease LCBs was of genetic nature. Arabidopsis contains three genes encoding SPT subunits: one LCB1 subunit (At4g36480) and two LCB2 subunits sharing 86\% identity: LCB2a (At5g23680) and LCB2b (At3g48780). LCB2a and LCB2b are functionally redundant when interacting with the LCB1 monomer to form SPT (Dietrich et al., 2008). Because $L C B 2 a$ gene expression is higher than $L C B 2 b$ in all plant organs analyzed thus far (Dietrich et al., 2008), we made the LCB2a contribution to LCB generation and accumulation in response to FB1 a focus of this study. We examined three independent T-DNA Arabidopsis mutants for LCB2a (Dietrich et al., 2008) in relation to FB1 response: lcb2a-1 (SALK 061472), lcb2a-2 (GABI 216-D07) and lcb2a-3 (SAIL_ 706-A05). On exposure to FB1, all mutants showed a slight decrease in growth, some chlorosis and flowering as a stress response (phenotype designated as FB1-resistant), but did not show the extensive tissue death seen in WT (Fig. 1b). These results indicate that these mutants may be useful as a tool to examine the molecular bases of PCD in comparison with WT seedlings which, on FB1 exposure, show an increase in LCB levels.

To substantiate the phenotypic cell death, we examined DNA fragmentation, a hallmark of PCD. Fragmented DNA was not detected in WT and $l c b 2 a-1$ control seedlings (Fig. 2a, sections 1-3, 7-9), but FB1-treated WT seedlings showed a higher proportion of free $3{ }^{\prime}-\mathrm{OH}$ groups, indicating fragmentation of DNA (Fig. 2 a, sections 4-6). By contrast, lcb2a-1 mutants showed few cells with DNA nicks (Fig. 2a, sections 10-12), implying that this mutant displays very low PCD features at the molecular level. These results were in agreement with the PCD appearance shown by the FB1-treated WT and mutant seedlings (Fig. 1b).

\section{PCD induced by LCB accumulation involves changes in the organization and integrity of organellar membranes}

In order to characterize the PCD features as a product of LCB buildup at a subcellular level, we used transmission electron microscopy (TEM) (Fig. 2b). WT seedlings showed typical cell morphology with large vacuoles restricting the cytoplasm to the periphery, and crescent-shaped 


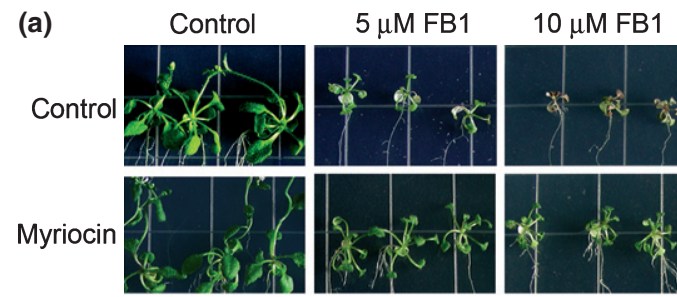

(b)
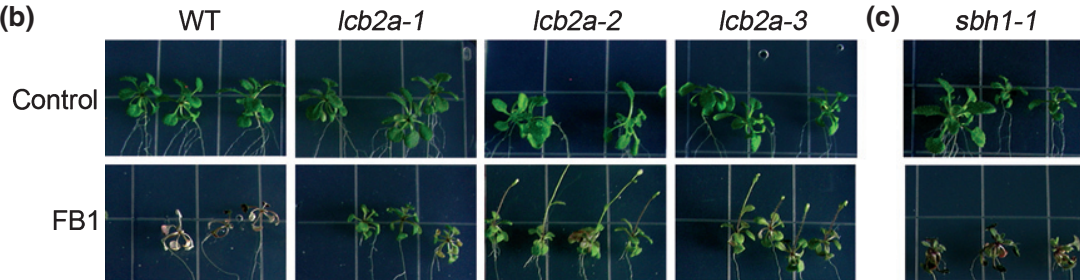

Fig. 1 Long chain base (LCB) accumulation induces cell death in Arabidopsis thaliana wild-type (WT) and sbh1-1 mutant seedlings, but not in Icb2a-1 mutant. (a) WT seedling phenotypes on fumonisin B1 (FB1) and myriocin treatments. WT Arabidopsis seedlings were exposed to 5 and $10 \mu \mathrm{M}$ FB1 without (top panel) or with (bottom panel) pre-incubation with $100 \mathrm{nM}$ myriocin for $5 \mathrm{~d}$. Phenotypes after $9 \mathrm{~d}$ of FB1 exposure are shown. (b) WT and Icb2a-1 seedling phenotypes on FB1 treatment. Phenotypes of WT Arabidopsis and three Icb2a mutant alleles exposed to control (top panel) or $10 \mu \mathrm{M}$ FB1 (bottom panel) for $9 \mathrm{~d}$. (c) sbh1-1 seedling phenotypes on FB1 treatment. Phenotypes of sbh1-1 mutant exposed to control (top panel) or $10 \mu \mathrm{M}$ FB1 (bottom panel) for $9 \mathrm{~d}$.

chloroplasts with well-formed thylakoid membranes (Fig. 2b, sections 1-3). By contrast, FB1-treated WT seedlings showed features of cells undergoing PCD: interiorized and rounded chloroplasts, indicating loss of tonoplast integrity (Fig. 2b, sections 4, 5); few starch grains (Yao \& Greenberg, 2006); and a remarkable disintegration of the thylakoids (Fig. 2b, section 5). In addition, we detected vesicles with inner structures resembling organellar debris, as reported in plant systems undergoing PCD (Liu Y et al., 2005; Mino et al., 2007) (Fig. 2b, section 6). The first visible ultrastructural signs of cell injury in WT seedlings occurred as early as $36 \mathrm{~h}$ post-FB1 treatment (Fig. S2).

In contrast with WT cells, disruption of the $L C B 2 a$ gene affected chloroplast morphology, in particular an increase in the size and number of rounded plastids (Fig. 2b, section 7). However, no dramatic changes were observed in thylakoid organization, starch granules or tonoplast integrity in the $l c b 2 a-1$ mutant exposed to control or FB1 conditions (Fig. 2b, sections 7-9 and 10-12, respectively). These results indicate that cell death promoted by an assumed LCB accumulation is coincident with DNA degradation and extensive organellar membrane damage. However, the FB1 resistance of the $l c b 2 a-1$ mutant was particularly intriguing, as no differences in growth and development, when compared with WT plants, were observed (Dietrich et al., 2008). Therefore, an approach to analyze the dynamics of endogenous LCBs was undertaken.

\section{Differential accumulation of LCBs during PCD elicitation in Arabidopsis seedlings}

To compare the FB1-induced accumulation of specific LCBs in WT and $l c b 2 a-1$ seedlings, we used a protocol that included the selective extraction of free LCBs and their subsequent separation and quantification by HPLC ESIMS/MS (Markham \& Jaworski, 2007). LCB profiling included the free nonphosphorylated and phosphorylated (LCB-P) forms of sphinganine (d18:0), 4-hydroxysphinganine (t18:0), 8-sphingenine (d18:1), 4,8-sphingadienine (d18:2) and 4-hydroxy-8-sphingenine (t18:1). Table S1 shows the determinations derived from five replicates for each treatment.

The levels of the 10 LCB species in seedlings grown under control conditions were very low during the $72-\mathrm{h}$ time span studied, both in WT and the lcb2a-1 mutant (Fig. 3, S3, Table S1), in agreement with the report by Markham et al. (2006). It was clearly observed that, on FB1 treatment, LCB levels increased as a function of time in absolute amounts, with higher levels in WT than in the lcb2a-1 mutant. d18:0, d18:0-P, t18:0 and t18:0-P showed major increases on FB1 treatment (Fig. 3, Table S1). The accumulation kinetics of $\mathrm{d} 18: 0$ and t18:0 in WT showed gradual and significant increases starting at $4 \mathrm{~h}$ and reaching similar absolute amounts at $72 \mathrm{~h}$ of FB1 exposure (Fig. 3a,b). At this time, although d18:0 had increased 619fold (compared with the WT, 0 -h value), the t18:0 increase was 113 -fold. With regard to d18:0-P and t18:0-P, a slight increase was observed from 4 to $12 \mathrm{~h}$, but a marked increase was attained at $72 \mathrm{~h}$ of FB1 exposure, corresponding to 7970-fold and 952-fold, respectively, when compared with the 0 -h value (Fig. 3c,d). The results of FB1 exposure of $l c b 2 a-1$ seedlings showed several differences from the WT treatment. There was no increase in d18:0 levels at $4 \mathrm{~h}$, and the levels accumulated between 8 and $12 \mathrm{~h}$ were very low when compared with the corresponding WT seedlings treated with the toxin; a high value was reached at $72 \mathrm{~h}$, when 
New

Phytologist
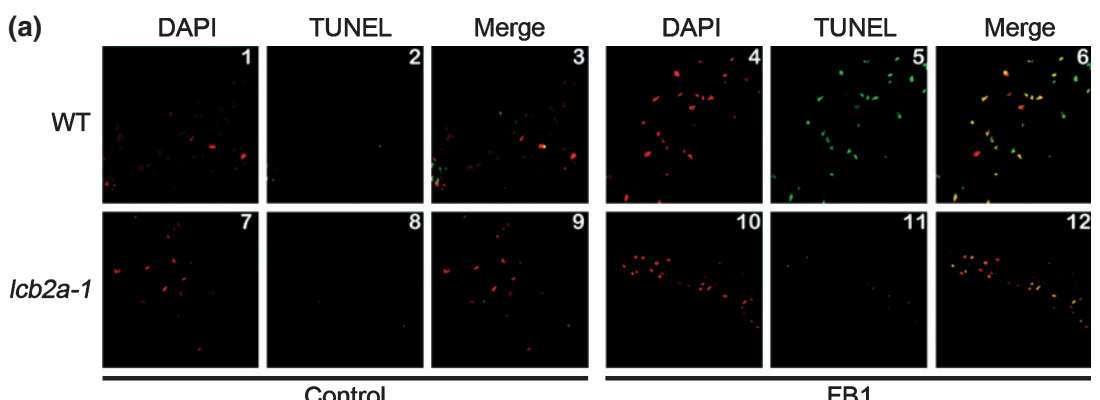

(b)
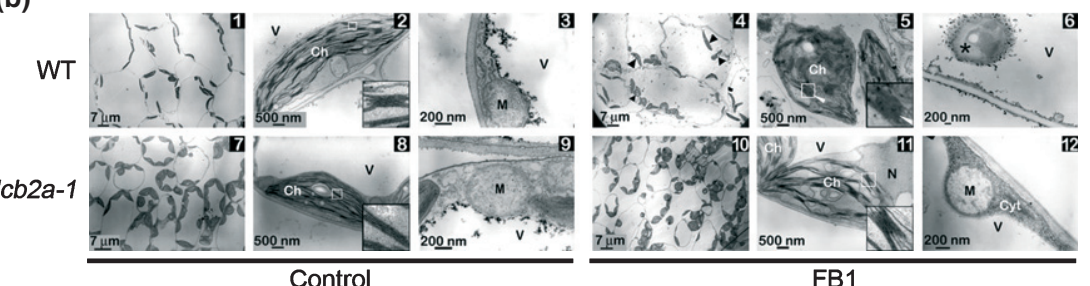

Fig. 2 Programmed cell death and the extensive endomembrane perturbations promoted by fumonisin B1 (FB1) are abated in Arabidopsis thaliana Icb2a-1 seedlings. (a) DNA fragmentation. This was measured in leaves from wild-type (WT) and /cb2a-1 seedlings not exposed (images 1-3 and 7-9, respectively) or exposed (images 4-6 and 10-12, respectively) to $10 \mu \mathrm{M}$ FB1 for $5 \mathrm{~d}$. The panels show nuclei stained with 4',6-diamidino-2-phenylindole (DAPI), the fluorescence of the nicked DNA in the nuclei and the resultant merged images. (b) Ultrastructural cellular analysis. This was performed in leaves of WT or /cb2a-1 seedlings not exposed (images 1-3 and 7-9, respectively) or exposed (images 4-6 and 10-12, respectively) to $10 \mu \mathrm{M}$ FB1 for $3 \mathrm{~d}$. Arrows show features with differences on FB1 treatment. Ch, chloroplast; Cyt, cytosol; M, mitochondria; N, nuclei; V, vacuole. Asterisk shows an autophagosome. Insets in images 2, 5, 8 and 11 show thylakoid enlargement of the corresponding image. Bars show the magnification.

the increment was 141-fold (compared with the lcb2a-1, 0 -h value) (Fig. $3 \mathrm{a}$ ). In contrast, t18:0 increased significantly from 4 to $72 \mathrm{~h}$ (41-fold at the latter time point), but at levels lower than those of the corresponding WT FB1treated samples (Fig. 3b). d18:0-P and t18:0-P attained similar levels to their nonphosphorylated forms, but with different increments (2186- and 452-fold, respectively) at 72 h (Fig. 3c,d).

From the four LCBs most affected by FB1 treatment, the clearest differences between WT and the $l c b 2 a-1$ mutant were in the d18:0 and d18:0-P contents, and in the earlier increase in $\mathrm{d} 18: 0$ in WT, suggesting that the timing and levels of $\mathrm{d} 18: 0$ and/or d18:0-P were the cause of the differences in the PCD phenotypes observed in WT and the lcb2a-1 mutant. The fact that d18:0 equalled t18:0 levels at $72 \mathrm{~h}$ in both WT and the lcb2a-1 mutant suggests that FB1 preferentially favors the endogenous accumulation of d18:0, although important increases in d18:0-P, t18:0 and t18:0-P were also found. In order to elucidate the function of t18:0 in the PCD elicited by FB1, we evaluated its contribution using a mutation in one of the two LCB hydroxylase genes $(S B H 1)$. It was found that the sbh1-1 mutant contained smaller amounts of trihydroxy-LCBs and elevated levels of total LCBs when compared with WT plants, although the $\mathrm{SBH} 2$ gene was functional (Chen et al., 2008). The rationale was that, if the trihydroxy-LCBs were main contributors to PCD, the sbh1-1 mutant would show FB1 resistance. The results in Fig. 1(c) show that
LCB-induced cell death in sbh1-1 mutants yields a similar appearance to WT plants, which were sensitive to FB1, implying that a decrease in trihydroxy-LCB levels does not inhibit PCD. This finding indicates that increased levels of trihydroxy-LCBs are not essential triggers in $\mathrm{PCD}$, and is consistent with the concept that dihydroxy-LCBs, such as $\mathrm{d} 18: 0$, may be the primary mediators of LCB-induced PCD.

\section{MPK6 participates in the PCD signaled by LCBs}

Recently, LCBs have been revealed as important players in HR-PCD, one of the most studied cases of PCD in plants (Takahashi et al., 2009; Lachaud et al., 2010; Peer et al., 2010) and in which MAPK cascades are involved. In order to evaluate the participation of MPKs in the signalling transduction pathway mediated by LCB accumulation, an in-gel MPK activity assay was performed in WT seedlings exposed to FB1. As shown in Fig. 4(a), FB1 induced an increase in the activity of an MPK of $c .46 \mathrm{kDa}$ throughout the interval of 15-90 min when compared with the control. From the MPKs in Arabidopsis, MPK6 and/or MPK3 have been repeatedly described as components of pathogen signaling pathways (Asai et al., 2002; Wang et al., 2007; Ren et al., 2008; Beckers et al., 2009). Thus, these two MPKs were selected in this study as possible candidates to be part of the LCB pathway that leads to PCD. Two Arabidopsis mutants were used: a T-DNA insertion line in 
(a)

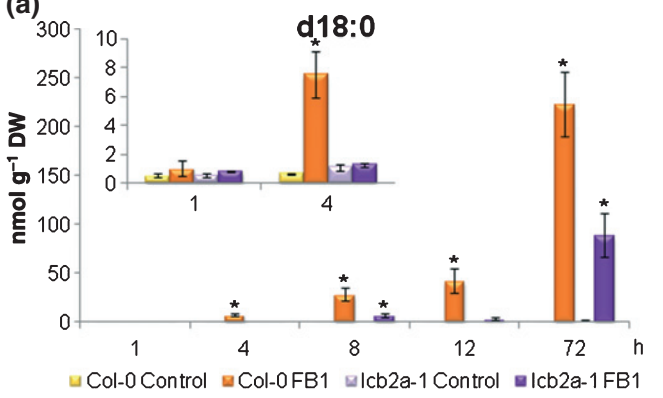

(b)

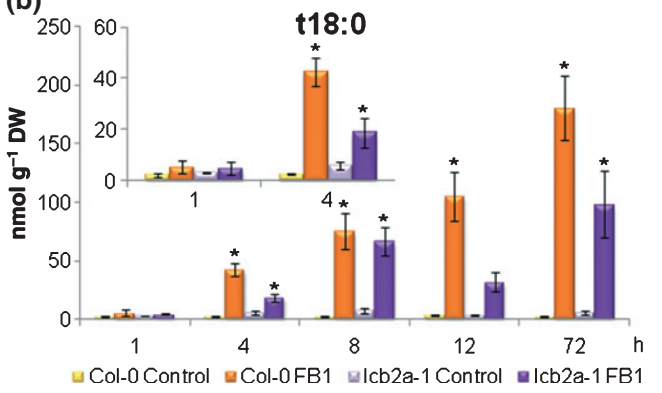

(c)

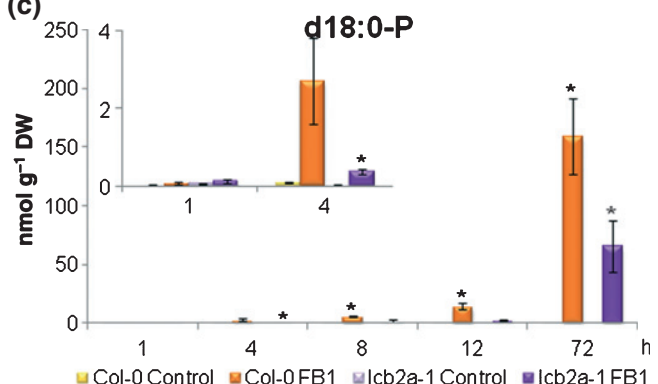

(d)

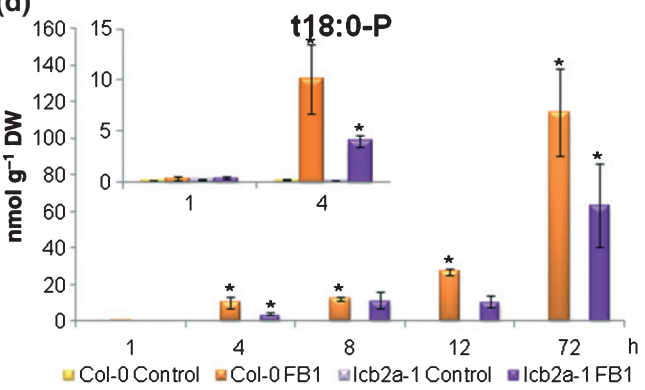

Fig. 3 The main free long chain bases (LCBs) accumulated under fumonisin B1 (FB1) treatment are d18:0 and t18:0 in their non- and phosphorylated forms, which are substantially decreased in the Arabidopsis thaliana Icb2a-1 mutant. The contents of free LCBs and phosphorylated LCBs (LCBs-P) in wild-type and Icb2a-1 seedlings (controls and exposed to FB1 for 1, 4, 8, 12 and 72 h) were determined after extraction, separation and identification by high-performance liquid chromatography coupled with electrospray ionization tandem mass spectrometry (HPLC ESI-MS/MS) as described in the Materials and Methods section. (a) Accumulation kinetics of d18:0 (sphinganine or dihydrosphingosine). (b) Accumulation kinetics of t18:0 (4-hydroxysphinganine or phytosphingosine). (c) Accumulation kinetics of d18:0-P (sphinganine 1-phosphate or dihydrosphingosine 1-phosphate). (d) Accumulation kinetics of t18:0-P (4-hydroxysphinganine 1-phosphate or phytosphingosine 1-phosphate). Values plotted are the averages of five independent determinations \pm standard error (SE). Asterisks represent statistically significant differences between FB1-treated seedlings and their corresponding controls at any given time; ${ }^{*}, P<0.05$. The corresponding average values of the five replicates \pm SE of the 10 LCBs are shown in Supporting Information Table S1. Insets show magnified sections (LCB determinations at 1 and $4 \mathrm{~h}$ ) of the full-scale graphs. The accumulation profiles of the rest of the LCBs are presented in Fig. S3.

the MPK6 gene and a MPK3 loss-of-function RNAi mutant, hereby referred to as $m p k 6$ and $m p k 3$, respectively. The $m p k 6$ mutant has been described as a null allele of MPK6 with a T-DNA insertion within the fourth exon. This mutant shows impairments in embryo, leaf and floral development (Bush \& Krysan, 2007; Wang et al., 2007) and in ethylene synthesis (Liu \& Zhang, 2004). mpk6 and mpk3 mutants showed undetectable transcript or protein levels (Fig. 4b). Mutant viability on FB1 treatment was then evaluated. The $m p k 6$ mutant exhibited resistance to FB1, similar to that observed in $l c b 2 a-1$ seedlings (Fig. 4c). An intermediate viability was seen in FB1-treated $m p k 3$ plants, with cell death symptoms less severe than in WT plants, but more pronounced than in mpk6 plants. These results strongly indicate the participation of MPK6 in the LCB-induced PCD.

To confirm that toxin resistance in the MPK mutants was related to the suppression of molecular PCD events, DNA fragmentation was also analyzed (Fig. 5a). FB1treated $m p k 6$ seedlings presented low levels of DNA fragmentation, similar to those exhibited by the $l c b 2 a-1$ mutant (Fig. 2a). By contrast, the mpk3 mutant presented more DNA fragmentation than mpk6, but less than WT. These results confirm that MPK6 participates in the pathway that triggers LCB-induced PCD and, moreover, suggest that both MPKs are not completely redundant in this PCD scheme.

As judged by TEM, the $m p k 6$ and $m p k 3$ mutants exhibited changes in chloroplast morphology (Fig. 5b, sections $1,2,7,8)$ and a larger number of mitochondria compared with the WT and $l c b 2 a-1$ lines (Fig. 5b, sections 3, 9). In addition, the FB1-treated mpk3 mutant showed several subcellular alterations: chloroplasts with smaller starch granules and disorganized grana with intact membranes (Fig. 5b, section 5); vacuoles with loss of turgor (Fig. 5b, section 5) that included membrane vesicles containing cytoplasmic materials and organelles resembling autophagosomes (Fig. 5b, section 6); and cytosol containing a large number of vesicles (Fig. 5b, section 6). By contrast, the FB1-treated $m p k 6$ seedlings only showed slight alterations in membrane and cell structure (Fig. 5b, sections 1012), and chloroplasts contained fewer but larger starch granules compared with untreated seedlings (Fig. 5b, section 10 vs section 7).

In order to confirm that LCBs and MPK6 were in the same signaling pathway leading to $\mathrm{PCD}$, several approaches 
New

Phytologist

(a)

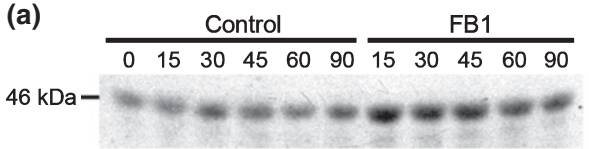

(b)

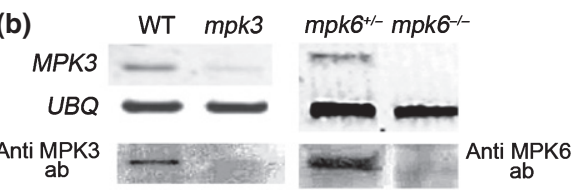

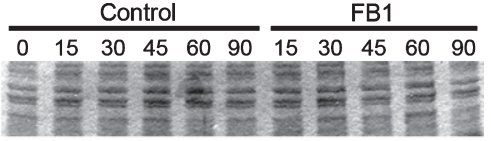

(c)

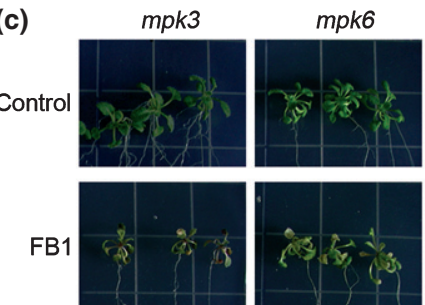

Fig. 4 The mpk6 mutant shows attenuated cell death under fumonisin B1 (FB1) treatment, and an in-gel assay shows a 46-kDa MPK activation by FB1 in wild-type (WT) seedlings. (a) In-gel MPK activity. WT seedlings were exposed to $10 \mu M$ FB1 at the indicated times expressed in minutes and their soluble fractions were used for in-gel assay of the MPK phosphorylating activity, as described in the Materials and Methods section. The left part of the panel shows the corresponding autoradiography and the right part shows the replicate gel stained with Coomassie blue as loading control. (b) Reverse transcription-polymerase chain reaction (RT-PCR) analysis of MPK3 and MPK6 transcripts. This was carried out in leaves of WT, $m p k 3$ and $m p k 6$ (heterozygous $/ m p k \sigma^{+/-}$and homozygous $/ m p k 6^{-/-}$) plants. Bottom panels show immunodetection of MPK3 and MPK6 proteins in WT and mpk3 and mpk6 mutants. (c) Phenotypes of mpk3 and mpk6 mutants. These are shown under control conditions (top panels) and on FB1 exposure (bottom panels) after $9 \mathrm{~d}$.

were examined. First, d18:0, t18:0 or d18:0-P was sprayed on WT, mpk3 and mpk6 seedlings (Fig. 6). Both WT and $m p k 3$ seedlings were highly susceptible to $\mathrm{d} 18: 0$ addition, whereas the $m p k 6$ mutant was resistant, as observed previously (Figs 1a, 4c). Treatment of WT and mpk3 with t18:0 and d18:0-P produced weak chlorosis, whereas $m p k 6$ seedlings showed a healthy phenotype similar to the control, indicating that $\mathrm{d} 18: 0$ was unable to launch the PCD pathway and that, even when $\mathrm{d} 18: 0$ was added to this mutant, the absence of MPK6 prevented cell death emergence. As a very important point, Fig. 6 shows that exogenously added $\mathrm{d} 18: 0$ induces a clear PCD phenotype in WT, which is not driven by t18:0 or d18:1-P. It should be noted that constant exposure of whole seedlings to FB1 leads to a generalized PCD that is accompanied by defense responses (Asai et al., 2000). To provide additional support for the hypothesis that LCBs and MPK6 are involved in the same route that orchestrates PCD, we used the $m k p 1$ mutant. MKP1 is an Arabidopsis cytosolic dual specificity phosphatase (DSP) that physically interacts with MPK3, MPK4 and MPK6 (Ulm et al., 2001, 2002), and is able to inactivate the MPK6 phosphorylated form (Ulm et al., 2002). Fig. 6 shows previously reported $m k p 1$ mutant features consisting of long pedicels and abnormal leaf development (Bartels et al., 2009). In addition, $m k p l$ is a mutant that constitutively shows defense responses, such as high levels of SA, camalexin and pathogenesis-related gene transcripts, producing an increase in disease resistance (Bartels et al., 2009). As observed in Fig. 6, supplementation of $m k p 1$ seedlings with d18:0 did not produce deleterious effects, suggesting that the upregulation of MPK6 and MPK3 activity enhanced defense reactions. The same resistance phenotype was observed in the $m p k 3 m k p 1$ double mutant and, to a minor extent, in $m p k 6 m k p 1$, suggesting that MPK6 is the main MPK needed for the establishment of the defense response. Treatments of $m k p 1, m p k 3 m k p 1$ and $m p k 6$ mkp1 mutants with t18:0 or d18:0-P did not produce significant changes, results that are in agreement with the phenotypes observed in WT and the single mutants.

In addition, to assess the association between LCB elevation and MPK6 activation, an in-gel MPK activity assay was performed (Fig. 7a). WT seedlings exposed to exogenously added FB1, d18:0 or t18:0 showed heavy myelin basic protein (MBP) labeling, corresponding to MPK6 activity and not to MPK3, as demonstrated by the molecular masses and activities shown in the $m p k 3$ and $m p k 6$ null mutants (Fig. 7a). Indeed, t18:0 showed the highest MPK6 activation, followed by $\mathrm{d} 18: 0$ and FB1. This result provides direct proof which clearly demonstrates that LCBs selectively use MPK6 and not MPK3 as their transducer towards PCD.

From a consideration of the reports that independently link MPK6 or LCBs to an HR-PCD (Menke et al., 2004; Ren et al., 2006; Takahashi et al., 2009), a supplementary strategy to support the involvement of LCBs and MPK6 in the same pathway leading to PCD was undertaken. We induced an HR-PCD through an exposure of WT, $l c b 2 a-1$ and $m p k 6$ seedlings to Pseudomonas syringae pv. tomato avrRpm1, a bacterial strain that is avirulent towards Arabidopsis and therefore produces a resistance response involving HR-PCD (Tao et al., 2003). Seedlings were pretreated with FB1 for $12 \mathrm{~h}$, and then exposed to the bacterial pathogen. Fig. 7(b) shows that FB1 treatment caused a decrease in bacterial proliferation in the WT and $l c b 2 a-1$ mutant seedlings when compared with the controls (nonFB1 treated). By contrast, the same treatment did not affect 

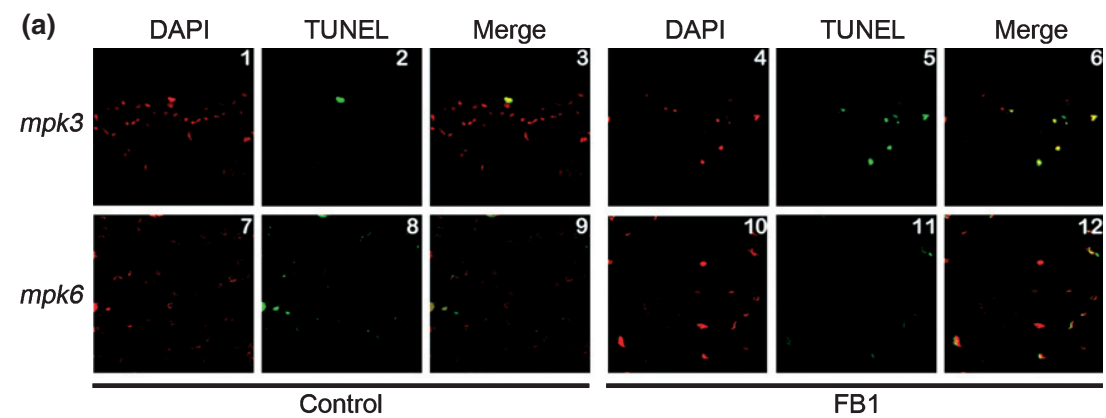

FB1
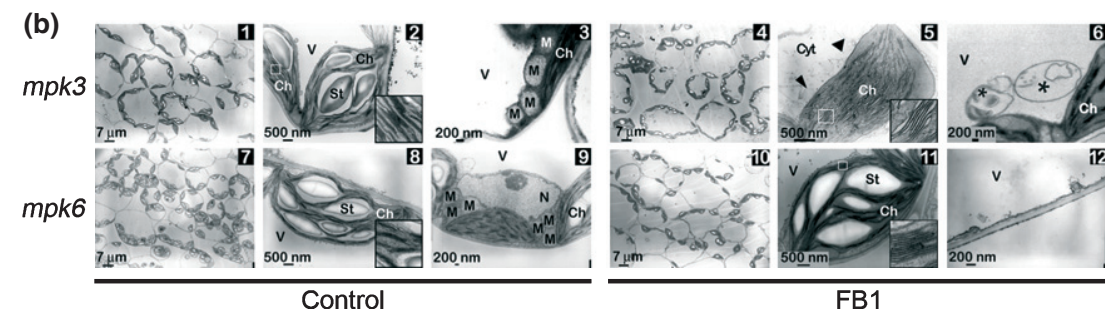

Fig. 5 The mpk6 mutant is unsuccessful in exhibiting cell features of programmed cell death when exposed to fumonisin B1 (FB1). (a) DNA fragmentation. This was measured by terminal deoxynucleotidyl transferase-mediated UTP nick end labeling (TUNEL) reaction in $m p k 3$ and mpk6 mutant seedlings not exposed (images 1-3 and 7-9, respectively) or exposed (images 4-6 and 10-12, respectively) to $10 \mu M$ FB1 for $5 \mathrm{~d}$. The panels show nuclei stained with 4',6-diamidino-2-phenylindole (DAPI), the fluorescence of the nicked DNA in the nuclei and the resultant merged images. (b) Ultrastructural cellular analyses. This was performed for leaves of $m p k 3$ or $m p k 6$ seedlings not exposed (images 1-3 and 7-9, respectively) or exposed (images 4-6 and 10-12, respectively) to $10 \mu \mathrm{M}$ FB1. Arrows show features modified after treatment with the toxin. Ch, chloroplast; Cyt, cytosol; M, mitochondria; N, nuclei; St, starch; V, vacuole. Asterisks indicate the presence of autophagosomes. Insets in 2, 5, 8 and 11 show thylakoid enlargement of the corresponding image. Bars show the magnification.

the bacterial proliferation in $m p k 6$ seedlings. These results indicate that an increase in LCBs and the intervention of MPK6 are required in the route that unchains PCD, but, more importantly, that the signaling route so configured decisively contributes to this strategy of defense against pathogens.

\section{Discussion}

\section{Role of specific SPT genes and specific LCBs in PCD elicitation}

Role of the $L C B 2 a$ gene In this study, we have reported that $L C B 2 a$ is required for the manifestation of PCD induced by FB1 based on the observation that, when the $L C B 2 a$ gene is absent, a significant attenuation in the levels of all analyzed LCB species is reached on FB1 exposure and very scarce signs of PCD are shown. Shi et al. (2007) found that a mutation in the $L C B 1$ gene contributed to lower LCB accumulation and therefore to FB1 resistance. This finding makes sense, as this gene is present in only one copy in Arabidopsis and the mutant used was only partially devoid of $L C B 1$ transcripts (indeed, total loss of this gene would have led to lethality; see Chen $e t$ al., 2006). Thus, as a result of this partial decrease in the $L C B 1$ gene, a diminished SPT activity produced minor increases in LCB on FB1 addition, and therefore a toxin-resistant phenotype. However, this is not the case in the present study, in which we evaluated one of the two $L C B 2$ isogenes. We can assign the functional significance of the $L C B 2 a$ gene in PCD elicitation to its higher transcription when compared with the $L C B 2 b$ gene (Dietrich et al., 2008). Consequently, the lcb2a-1 mutant, which displays an FB1-resistant phenotype, maintains an SPT activity only through the formation of an LCB1/LCB2b dimer which, as a result of lower $L C B 2 b$ expression, may lead to an inability to achieve high levels of LCBs. The majority of SPT complexes that generate LCBs in WT plants are composed of LCB1/LCB2a subunits, and therefore are committed to the increased formation of LCBs that mediate PCD, supporting the role of SPT as a PCD regulator in Arabidopsis. The nonfunctional redundancy of the Arabidopsis LCB $2 \mathrm{a} / \mathrm{b}$ subunits has been described recently in vertebrates, which contain an alternative SPT subunit (SPTCL3) analog to LCB2 that uses shorter acyl chain-CoA to synthesize C-16 sphinganine (Hornemann et al., 2009). The importance of the differential expression of SPT genes under stress conditions has recently been illustrated in the case of the $L C B 2$ gene in Nicotiana benthamiana, whose overexpression is induced by the nonhost pathogen Pseudomonas cichorii, enhancing PCD (Takahashi et al., 2009).

In addition to the contribution of the LCB1/LCB2aSPT complex to the high accumulation of d18:0 (sphinganine), FB1 could be exerting a differential inhibition on the two proposed ceramide synthase (CS) activities: CSI (which uses $\mathrm{d} 18: 0$ as substrate) would be sensitive to FB1, whereas 
New

Phytologist

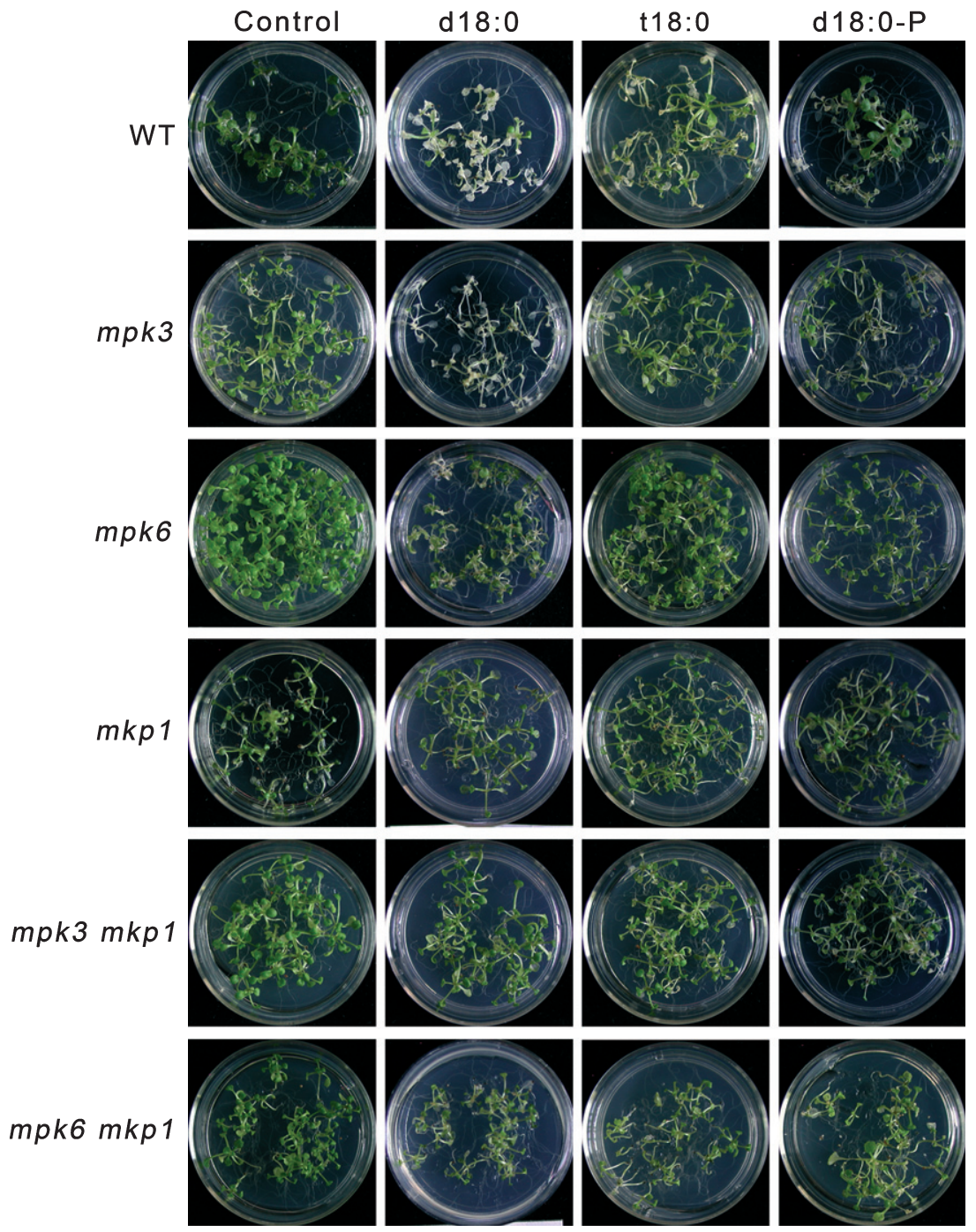

Fig. 6 MPK6 operates downstream of long chain bases (LCBs) in the fumonisin B1 (FB1)-induced programmed cell death through an activation mainly mediated by d18:0. Wild-type (WT), mpk3, mpk6, mkp1, mpk3 mkp1 and mpk6 mkp1 mutant seedlings were exposed to exogenous d18:0 $(5 \mu \mathrm{M})$, t18:0 $(5 \mu \mathrm{M})$, d18:0-P $(5 \mu \mathrm{M})$ or 0.04\% Silwet (control) solutions under continuous light. Photographs were taken after $4 \mathrm{~d}$ of exposure. (See the Materials and Methods section for experimental details.)

CSII (which uses t18:0) (Chen et al., 2009) would show less FB1 inhibition. Therefore, the increase in d18:0 concentration, acting as a second messenger in the PCD route, is generated by a complex physiological regulation of different enzymatic reactions involving sphinganine metabolism.

Role of $\mathbf{d} 18: 0$ We postulate that $\mathrm{d} 18: 0$ is the main LCB responsible for signaling in the LCB-induced PCD on the basis of three lines of evidence. First, the correlation between the high levels reached by this LCB in WT seedlings on FB1 treatment and the PCD phenotype, a correlation that is not achieved by the lcb2a-1 mutant. Second, the addition of exogenous d18:0 to WT seedlings, which results in a clear PCD phenotype, is barely shown by t18:0. Third, the Arabidopsis double mutant sbh1 sbh2, which is defective in the two LCB hydroxylases and shows a remarkable increase in dihydroxylated LCBs, manifests necrotic spots and premature death (Chen et al., 2008), supporting the concept that the accumulation of sphinganine (d18:0) produces PCD. This interpretation is strengthened by a very recent study reporting that the mutant sbh1-1, which shows decreased levels of t18:0, but increased total LCB levels, fails to show susceptibility to infection by Pseudomonas syringae in an HR-PCD model (Peer et al., 2010). Moreover, these authors provide evidence suggesting that the sustained increase in t18:0 on avirulent bacterial infection originates from hydroxylase activity on the de novo-synthesized $\mathrm{d} 18: 0$, as ceramide species seem unchanged. In this sense, our data confirm this view and indicate that the $\mathrm{LCB}$ species involved in FB1 
(a)
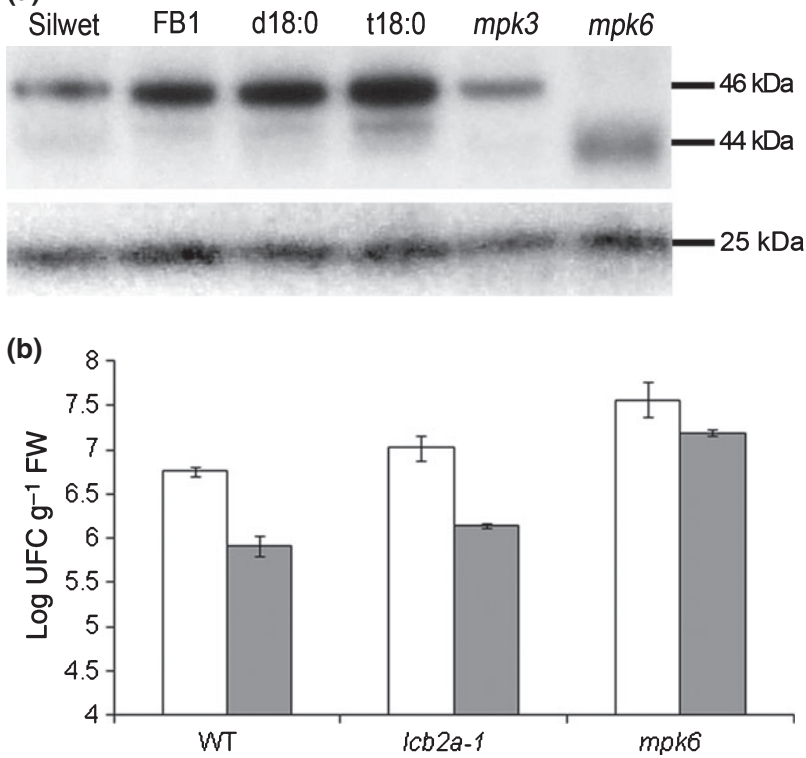

Fig. 7 MPK6 is activated by exogenous long chain bases (LCBs) and this association is required to express the hypersensitive response programmed cell death used as a defense strategy against pathogens. (a) In-gel MPK activity. Protein extracts from wild-type (WT) seedlings exposed to exogenous fumonisin B1 (FB1) (10 $\mu \mathrm{M})$, d18:0 $(5 \mu \mathrm{M})$, t18:0 $(5 \mu \mathrm{M})$ or Silwet $(0.04 \%$ as control) solutions for $15 \mathrm{~min}$ were used for in-gel activity assay. Protein extracts from $m p k 3$ and $m p k 6$ mutants were included in the assay to identify the activity of MPK3 and MPK 6 in the gel. (See the Materials and Methods section for experimental details.) Both parts of the panel correspond to autoradiography of the gel. The bottom part includes an unspecific phosphorylated band as a loading control. (b) Bacterial growth in planta. WT and Icb2a-1 or mpk6 mutant seedlings were transferred to a medium without (open bars) or with $10 \mu \mathrm{M}$ FB1 (closed bars) for $12 \mathrm{~h}$. Then, the seedlings were challenged with a suspension of Pseudomonas syringae pv. tomato avrRpm1 at a concentration of 107 colony-forming units. After $48 \mathrm{~h}$, sampling was performed in triplicate to determine bacterial growth in the seedlings. Independent experiments were carried out at least three times. (See the Materials and Methods section for experimental details.).

PCD elicitation, and which evoke a defense reaction, are de novo synthesized, indicating that these signaling species are not derived from the degradation of complex lipids, as is the case for the classic signaling mediated by lyso-glycerophospholipids (Wang, 2004). Endorsing our interpretation of d18:0 but not t18:0 as an operator in signaling towards PCD, we cite the work of Lachaud et al. (2010), in which the external administration of d18:0 to tobacco BY2 cells induced an increase in $\mathrm{Ca}^{2+}$ concentration in the cytosol, and later in the nucleus, which was essential for the expression of an apoptotic-like PCD. [Correction added after online publication 27 April 2011: the preceding sentence has been altered to correctly indicate the work of Lachaud et al. (2010).]

Despite the aforementioned arguments in favor of $\mathrm{d} 18: 0$ as a main contributor to PCD elicitation, the observed high accumulation of $t 18: 0$ and the phosphorylated forms of d18:0 and t18:0 under FB1 exposure prompted us to explore their role in PCD unchaining. With regard to the participation of t18:0 in PCD, the LCB increases in WT FB1-treated seedlings were lower than those of $\mathrm{d} 18$ :0 associated with PCD expression, weakening the possible cardinal role of t18:0 in PCD elicitation. In addition, we found that the sbh1-1 hydroxylase mutant (Chen et al., 2008) was able to express an FB1-induced PCD phenotype, indicating that t18:0 is not the leading intermediate of PCD. Supporting this interpretation, we must mention the results from Chen et al. (2008) with the Arabidopsis sbh1 sbh2 mutant, which is enriched in dihydroxylated LCB species, but contains no trihydroxylated LCBs, and nonetheless shows spontaneous PCD lesions, implying that high levels of trihydroxylated LCBs do not elicit PCD by themselves.

We cannot discount a role of $\mathrm{d} 18: 0$ and t18:0 phosphoryated forms in PCD, as we determined that $\mathrm{d} 18: 0-\mathrm{P}$ and t18:0-P increased in WT seedlings to very important levels after 72 h of FB1 exposure (c. 8000- and 1000-fold, with respect to the 0 -h value, respectively). However, the external addition of d18:0-P showed meager PCD symptoms. It must be acknowledged that, in plants, most of the available evidence on LCBs as second messengers has been reported for the phosphorylated LCB forms. Stomatal closure under drought conditions is driven by the activity of ion channels in the plasma membranes of guard cells (Schroeder et al., 2001), and is induced by $\mathrm{Ca}^{2+}$ mobilization and elevation in d18:1-P levels (a desaturated form of d18:0) in Commelia communis ( $\mathrm{Ng}$ et al., 2001). However, Michaelson et al. (2009) reported that the ablation of the $\Delta 4$-desaturase gene did not affect guard cell closure in Arabidopsis. Thus, t18:0$\mathrm{P}$ could fulfill this role, as t18:0 is a substrate of sphingosine kinase (SphK) (Coursol et al., 2005; Worrall et al., 2008). This is an LCB kinase involved in abscisic acid (ABA) signaling of stomatal closure (Coursol et al., 2005; Worrall et al., 2008), which could generate d18:0-P in the case of BY2 cells (Lachaud et al., 2010) or t18:0-P in the case of guard cells (Coursol et al., 2005; Worrall et al., 2008), and could potentially trigger $\mathrm{Ca}^{2+}$ elevation. In animal cells, SphK2, a nuclear SphK, has a proapoptotic role, producing LCBs-P (Maceyka et al., 2005). The importance of the maintenance of low LCBs-P levels has been manifested by the FB1 hypersensitivity of the $L C B$ lyase mutant in Arabidopsis (Tsegaye et al., 2007).

\section{MPK6 as a transducer in PCD orchestrated by LCBs}

In this work, we have demonstrated that MPK6 has a regulatory role in PCD induced by LCBs. We have provided four lines of experimental evidence to support this finding. First, our results with the $m p k 6$ mutants showed that LCBinduced PCD requires the mediation of MPK6 downstream of LCB accumulation, as the elevation of endogenous LCBs induced by FB1 did not produce severe changes in the 
mpk6 seedling phenotype. Second, the exogenous addition of $\mathrm{d} 18: 0$ was unable to induce PCD in $m p k 6$ seedlings. Third, MPK6 showed an activation of its MBP phosphorylating capacity, either by the endogenous increase in LCBs driven by FB1 exposure or by the exogenously supplied LCBs. Fourth, the association described between MPK6 and HR-PCD manifestation as part of the defense reactions against pathogens was abolished in the mpk6 mutant, implying that this association is part of a transducing pathway that has the aim of launching a PCD process as a method to contend with pathogen spreading.

Our shortest time of LCB determination on seedling exposure to FB1 was $1 \mathrm{~h}$ and significant increases in LCBs were reached in WT and $l c b 2 a-1$ seedlings at $4 \mathrm{~h}$. However, the rapid MPK6 activation (15 min) observed on exogenous LCB or FB1 addition suggests that this MAPK cascade is very sensitive to small changes in the endogenous levels of LCBs that may not be well detected by HPLC ESI-MS/MS. It is noteworthy to point out that such rapid MPK6 activation in response to supplemented LCBs is coincident with the 12-20-min maximal nuclear $\mathrm{Ca}^{2+}$ accumulation promoted by sphinganine addition to tobacco cells (Lachaud et al., 2010). These rapid responses are common in signaling molecules, such as phosphatidic acid species, which increase within 10 min of ABA addition (Zhang et al., 2009).

With the exclusion of LCBs, MPK6 upstream elements in this PCD model are unknown. As many possible activators have been proposed, these may include transducers, such as proteins/kinases, carrying pleckstrin homology domains that can recognize FB1 or LCBs (Wang et al., 1991; Popescu et al., 2009). G proteins are among the possible initial signaling factors as, in rice, the Arabidopsis MPK6 ortholog that is induced by a sphingolipid elicitor isolated from a blast rice fungus is regulated upstream by a heterotrimeric G protein and a small GTPase (Lieberherr et al., 2005). In addition, d18:1P, produced by SphK, is upstream of $\mathrm{G} \alpha$ during the $\mathrm{ABA}$ signaling that regulates the stomatal aperture in Arabidopsis guard cells (Coursol et al., 2003; Pandey \& Assmann, 2004).

With regard to MPK6 downstream elements in the LCB-PCD transduction pathway, Asai et al. (2000) reported the requirement of $S A$, jasmonic acid (JA) and ethylene in the FB1-induced PCD. In addition, our results showing the low expression of PCD induced by d18:0 in the mpk1 mutant, with increased SA levels (Bartels et al., 2009), suggest that SA generation is downstream of MPK6, as the $m p k 6 m k p 1$ mutant was more susceptible to LCB action than the $m k p 1$ and/or $m p k 3 m k p 1$ mutant.

MPK6 is associated with the trans-Golgi network (TGN) and plasma membrane, but absent in the endoplasmic reticulum in Arabidopsis thaliana roots (Müller et al., 2010). The fact that we found MPK6 activation on FB1 exposure raises the possibility that, in mesophyll cells, MPK6 is associated with membrane compartments with high LCB contents, in order to facilitate its activation by the corresponding upstream elements in the MAPK cascade.

Another important result was that MPK6, but not MPK3, was essential to LCB-induced PCD. This finding is in contrast with multiple other cases in which both kinases, which are very close homologs, show high functional redundancy (Colcombet \& Hirt, 2008). These kinases may be differentially expressed under circumstances that involve specific requirements, such as time of response, cellular type or abundance. For example, MPK3, but not MPK6, regulates either pathogen-induced or $\mathrm{H}_{2} \mathrm{O}_{2}$-induced stomatal closure (Gudesblat et al., 2007) and shows transcriptional regulation (Walley et al., 2007; Pitzschke et al., 2009). By contrast, MPK6, but not MPK3, is essential in gametogenesis and the development of reproductive organs, but no control of its gene expression has been found (Bush \& Krysan, 2007). However, although activation of both MPK3 and MPK6 is involved in cases of immunity-related PCD as previously reported (Ren et al., 2006), this functional redundancy of MPK6 and MPK3 does not apply to the route of PCD instrumented by LCB elevation, same that selectively requires MPK6, as derived from our experiments.

\section{Cell features of LCB-induced PCD}

The features of cells undergoing PCD as a consequence of LCB accumulation are practically unknown. We observed DNA fragmentation and the loss of integrity of organellar membranes. The incorporation of LCB overload and the decrease in complex sphingolipids may account for membrane disruption; however, oxidative stress may also be responsible for some PCD-driven cell perturbations (Levine et al., 1994), as it has been reported that LCB-induced PCD is mediated by downstream $\mathrm{H}_{2} \mathrm{O}_{2}$ generation (Shi et al., 2007).

It has been reported that the overexpression of $\mathrm{NtMEK}^{\mathrm{DD}}$ (a MAPKK) in tobacco leads to a prolonged activation of SIPK, WIPK and Ntf4, inhibiting $\mathrm{CO}_{2}$ fixation and generating excess energy excitation (Liu et al., 2007). Mutations in MPK3 and MPK6 seem to be important in starch metabolism, as mutants of these kinases showed chloroplasts with starch granules of large dimensions. As SIPK and WIPK are the tobacco orthologs of Arabidopsis MPK6 and MPK3, respectively, it is possible that a loss of function of these MPKs in Arabidopsis favors carbon fixation, increasing carbohydrate synthesis and starch deposition.

The observed subcellular features were consistent with autophagic cell death, as reported for FB1-induced PCD in Arabidopsis, and which has been related to the activity of vacuolar processing enzymes (VPEs) (Kuroyanagi et al., 2005). It is possible that some of the effects of MPK6 are related to the activity of VPEs, as mutations in the four $V P E$ genes preserve the membrane integrity of FB1-treated cells (Kuroyanagi et al., 2005). 


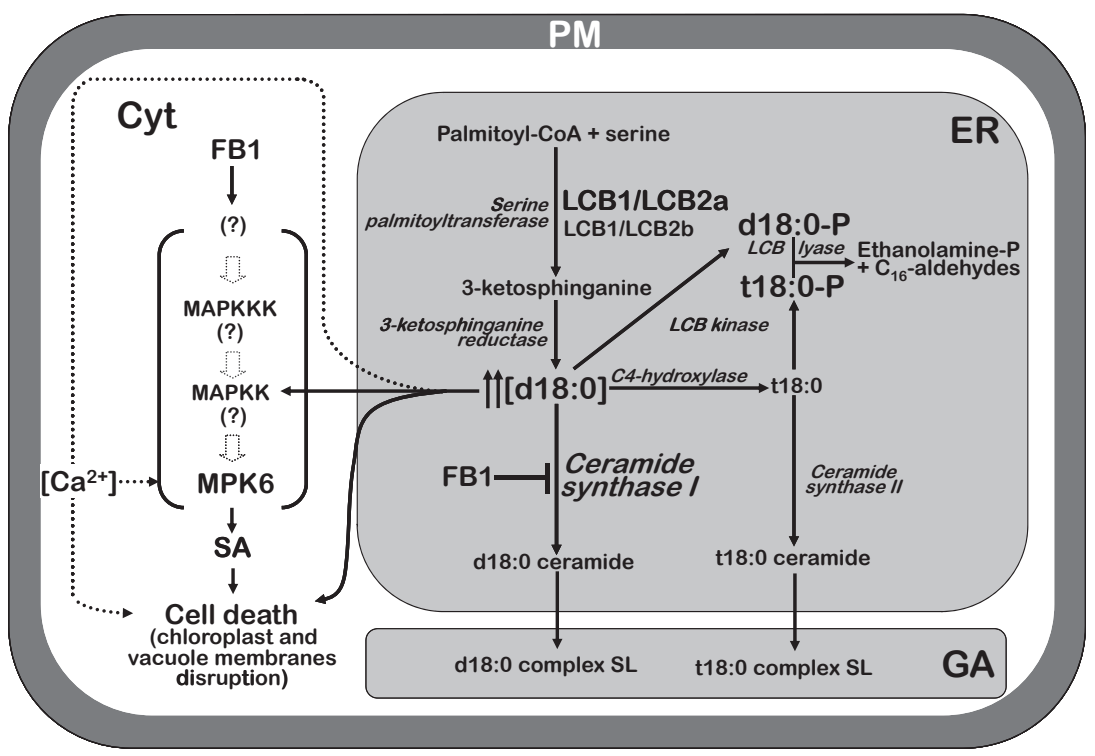

Fig. 8 Model considering long chain bases (LCBS) and MPK6 as components of the transduction pathway leading to programmed cell death in Arabidopsis. Dashed line indicates possible points of $\mathrm{Ca}^{2+}$ action. [Correction added after online publication 27 April 2011: the position of the dashed line has been altered to originate from d18:0 and not d18:0-P according to the findings of this article and those of Lachaud et al. (2010).] The names of the enzymes of sphingolipid synthesis are given in italics. ER, endoplasmic reticulum; FB1, fumonisin B1; GA, Golgi apparatus; Nuc, nuclear; PM, plasma membrane; SA, salicylic acid; SL, sphingolipids.

The absence of PCD observed in the mpk6 mutant reveals that LCB accumulation per se is not sufficient to promote the breakdown of DNA and cell membranes, as MPK6 seems to be required. A feasible explanation for this finding is that MPK6 is responsible for reactive oxygen species generation at levels leading to PCD. This is consistent with the available evidence that associates MPK6, reactive oxygen species formation and PCD (Liu et al., 2007).

\section{General model}

LCB-induced PCD in Arabidopsis seedlings results from three factors: the activity of the LCB1/LCB2a-SPT complex, the increase in sphinganine $(\mathrm{d} 18: 0)$ and the activation of MPK6 (Fig. 8). Sphinganine accumulation is favored by the efficient de novo synthesis of its precursor by the LCB1/LCB2a-SPT complex and by the predominant inhibition of FB1 over CSI. The elevation of LCBs as a second messenger targets a transducer element that leads to downstream MAPK activation through MPK6, amplifying death signals. An additional effect of FB1 on the same transducer element reached by $\mathrm{d} 18: 0$ cannot be excluded, because of the structural similarities between the two molecules (as shown for CS that recognizes both FB1 and dihydrosphinganine; see Wang et al., 1991). The participation of enzymes that use $\mathrm{d} 18: 0$ or its derivatives as substrates is essential for a tight and differential regulation of LCB levels. The model includes the role of $\mathrm{Ca}^{2+}$ as an inducing PCD factor in the signaling pathway unchained by LCB elevation (Lachaud et al., 2010) and SA generation downstream of LCB signaling (Fig. 8).
A considerable body of evidence has confirmed the participation of MPK6 in HR-PCD. More recent experimentation has associated sphingolipids with PCD signaling. In the present work, our results indicate that both MAP cascades and sphingolipids are transducers in the same PCD pathway, and that this operates as one of the strategies used as defense against pathogens (HR-PCD). In addition, evidence has been provided linking the participation of specific LCBs, SPT complexes and MPK forms in LCB-induced PCD, contributing to the deciphering of cell death transduction pathways in plants.

\section{Acknowledgements}

We thank M. C. Enríquez-Arredondo for excellent technical assistance and R. Paredes-Díaz for his expertise in TEM sample preparation. We appreciate the contribution of L. Fabila-Ibarra to glasshouse work, Dr L. F. GarcíaJiménez for the analysis of early TEM samples, Drs R. Cahoon (University of Nebraska-Lincoln, NE, USA), D. González-Halphen and M. Macías (Instituto de Fisiología Celular, UNAM, México) for critical comments, Drs M. A. González Besteiro and R. Ulm (University of Geneva, Switzerland) for the $m k p 1, m p k 3 m k p 1$ and $m p k 6 m k p 1$ mutants, and Dr D. F. Klessig for the initial gift of antiMPK6 antibodies and mpk6 mutant seeds. We thank BBA. E. Mendoza and M. Raya from Chemtura Corporation, México, for the gift of Silwet L-77. This work was supported by grants from Dirección General de Asuntos del Personal Académico (DGAPA), Universidad Nacional 
Autónoma de México (UNAM) (IN207806, IN211409, IN21768) and Consejo Nacional de Ciencia y Tecnología (CONACyT) (101521), Mexico. M.S.G. received CONACyT (185332) and DGAPA (IN211409) PhD Fellowships.

\section{References}

Asai T, Stone JM, Heard JE, Kovtun Y, Yorgey P, Sheen J, Ausubel FM. 2000. Fumonisin B1-induced cell death in Arabidopsis protoplasts requires jasmonate-, ethylene-, and salicylate-dependent signaling pathways. Plant Cell 12: 1823-1836.

Asai T, Tena G, Plotnikova J, Willmann MR, Chiu W-L, Gomez-Gomez L, Boller T, Ausubel FM, Sheen J. 2002. MAP kinase signalling cascade in Arabidopsis innate immunity. Nature 415: 977-983.

Bartels S, Anderson JC, González Besteiro MA, Carreri A, Hirt H, Buchala A, Métraux J-P, Peck SC, Ulm R. 2009. MAP kinase phosphatase 1 and protein tyrosine phosphatase 1 are repressors of salicylic acid synthesis and SNC1-mediated responses in Arabidopsis. Plant Cell 21: 2884-2897.

Beckers GJ, Jaskiewicz M, Liu Y, Underwood WR, He SY, Zhang S, Conrath U. 2009. Mitogen-activated protein kinases 3 and 6 are required for full priming of stress responses in Arabidopsis thaliana. Plant Cell 21: 944-953.

Brodersen P, Petersen M, Pike HM, Olszak B, Skov S, Odum N, Jørgensen LB, Brown RE, Mundy J. 2002. Knockout of Arabidopsis ACCELERATED-CELL-DEATH11 encoding a sphingosine transfer protein causes activation of programmed cell death and defense. Genes and Development 16: 490-502.

Bush SM, Krysan PJ. 2007. Mutational evidence that Arabidopsis MAP kinase MPK6 is involved in anther, inflorescence, and embryo development. Journal of Experimental Botany 58: 2181-2191.

Chen M, Cahoon EB, Saucedo-García M, Plasencia J, Gavilanes-Ruíz M. 2009. Plant sphingolipids: structure, synthesis and function. In: Wada H, Murata N, eds. Lipids in photosynthesis: essential and regulatory functions. Dordrecht, the Netherlands: Springer, 77-115.

Chen M, Han G, Dietrich CR, Dunn TM, Cahoon EB. 2006. The essential nature of sphingolipids in plants as revealed by the functional identification and characterization of the Arabidopsis LCB1 subunit of serine palmitoyltransferase. Plant Cell 18: 3576-3593.

Chen M, Markham JE, Dietrich CR, Jaworski JG, Cahoon EB. 2008. Sphingolipid long-chain base hydroxylation is important for growth and regulation of sphingolipid content and composition in Arabidopsis. Plant Cell 20: 1862-1878.

Colcombet J, Hirt H. 2008. Arabidopsis MAPKs: a complex signaling network involved in multiple biological processes. Biochemical Journal 413: 217-226.

Coursol S, Fan L-M, Le Stunff H, Spiegel S, Gilroy S, Assmann SM. 2003. Sphingolipid signalling in Arabidopsis guard cell involves heterotrimeric G proteins. Nature 423: 651-654.

Coursol S, Le Stunff H, Lynch DV, Gilroy S, Assmann SM, Spiegel S. 2005. Arabidopsis sphingosine kinase and the effects of phytosphingosine-1-phosphate on stomatal aperture. Plant Physiology 137: 724-737.

Desikan R, Hancock JT, Ichimura K, Shinozaki K, Neill SJ. 2001. Hairpin induces activation of the Arabidopsis mitogen-activated protein kinases AtMPK4 and AtMPK6. Plant Physiology 126: 1579-1587.

Dietrich CR, Han G, Chen M, Berg RH, Dunn TM, Cahoon EB. 2008. Loss-of-function mutations and inducible RNAi suppression of Arabidopsis LCB2 genes reveal the critical role of sphingolipids in gametophytic and sporophytic cell viability. Plant Journal 54: 284-298.

Gable K, Han G, Monaghan E, Bacikova D, Natarajan M, Williams R, Dunn TM. 2002. Mutations in the yeast $L C B 1$ and $L C B 2$ genes, including those corresponding to the hereditary sensory neuropathy type I mutations, dominantly inactivate serine palmitoyltransferase. Journal of Biological Chemistry 277: 10194-10200.

Greenberg JT. 1996. Programmed cell death: a way of life for plants. Proceedings of the National Academy of Sciences, USA 93: 12094-12097.

Gudesblat GE, Iusem ND, Morris PC. 2007. Guard cell-specific inhibition of Arabidopsis MPK3 expression causes abnormal stomatal responses to abscisic acid and hydrogen peroxide. New Phytologist 17: 713-721.

Hannun YA, Obeid LM. 2008. Principles of bioactive lipid signalling: lessons from sphingolipids. Nature Reviews Molecular Cell Biology 9: $139-150$.

Hornemann T, Penno A, Rutti MF, Ernst D, Kivrak-Pfiffner F, Rohrer L, von Eckardstein A. 2009. The SPTLC3 subunit of serinepalmitoyltransferase generates short chain sphingoid bases. Journal of Biological Chemistry 284: 26322-26330.

Kuroyanagi M, Yamada K, Hatsugai N, Kondo M, Nishimura M, HaraNishimura I. 2005. Vacuolar processing enzyme is essential for mycotoxin-induced cell death in Arabidopsis thaliana. Journal of Biological Chemistry 280: 32914-32920.

Lachaud C, Da Silva D, Cotelle V, Thuleau P, Xiong TC, Jauneau A, Brière C, Graziana A, Bellec Y, Faure JD et al. 2010. Nuclear calcium controls the apoptotic-like cell death induced by d-erythro-sphinganine in tobacco cells. Cell Calcium 47: 92-100.

Levine A, Tenhaken R, Dixon R, Lamb C. 1994. $\mathrm{H}_{2} \mathrm{O}_{2}$ from the oxidative burst orchestrates the plant hypersensitive disease resistance response. Cell 79: 583-593.

Liang H, Yao N, Song JT, Luo S, Lu H, Greenberg JT. 2003. Ceramides modulate programmed cell death in plants. Genes and Development 17: 2636-2661.

Lieberherr D, Thao NP, Nakashima A, Umemura K, Kawasaki T, Shimamoto K. 2005. A sphingolipid elicitor-inducible mitogenactivated protein kinase is regulated by the small GTPase OsRac1 and heterotrimeric G-protein in rice. Plant Physiology 138: 1644-1652.

Liu K, Zhang X, Sumanasekera C, Lester RL, Dickson RC. 2005. Signalling functions for sphingolipid long-chain bases in Saccharomyces cerevisiae. Biochemical Society Transactions 33: 1170-1173.

Liu Y, Ren D, Pike S, Pallardy S, Gassmann W, Zhang S. 2007. Chloroplast-generated reactive oxygen species are involved in hypersensitive response-like cell death mediated by a mitogen-activated protein kinase cascade. Plant Journal 51: 941-954.

Liu Y, Schiff M, Czymmek K, Tallóczy Z, Levine B, Dinesh-Kumar SP. 2005. Autophagy regulates programmed cell death during the plant innate immune response. Cell 121: 567-577.

Liu Y, Zhang S. 2004. Phosphorylation of 1-aminocyclopropane-1carboxylic acid synthase by MPK6, a stress-responsive mitogen-activated protein kinase, induces ethylene biosynthesis in Arabidopsis. Plant Cell 16: 3386-3399.

Maceyka M, Sankala H, Hait NC, Le Stunff H, Liu H, Toman R, Collier C, Zhang M, Satin LS, Merrill AH Jr et al. 2005. SphK1 and SphK2, sphingosine kinase isoenzymes with opposing functions in sphingolipid metabolism. Journal of Biological Chemistry 280: 37118-37129.

Markham JE, Jaworski JG. 2007. Rapid measurement of sphingolipids from Arabidopsis thaliana by reversed-phase high-performance liquid chromatography coupled to electrospray ionization tandem mass spectrometry. Rapid Communications in Mass Spectrometry 21: 1304-1314

Markham JE, Li J, Cahoon EB, Jaworski JG. 2006. Separation and identification of major plant sphingolipid classes from leaves. Journal of Biological Chemistry 281: 22684-22694.

Menke FL, van Pelt JA, Pieterse CM, Klessig DF. 2004. Silencing of the mitogen-activated protein kinase MPK6 compromises disease resistance in Arabidopsis. Plant Cell 16: 897-907. 
Merrill AH Jr, Sullards MC, Wang E, Voss KA, Riley RT. 2001. Sphingolipid metabolism: roles in signal transduction and disruption by fumonisins. Environmental Health Perspectives 109: 283-289.

Michaelson LV, Zäuner S, Markham JE, Haslam RP, Desikan R, Mugford S, Albrecht S, Warnecke D, Sperling P, Heinz E et al. 2009. Functional characterization of a higher plant sphingolipid $\Delta 4$ desaturase: defining the role of sphingosine and sphingosine-1phosphate in Arabidopsis. Plant Physiology 149: 487-498.

Mino M, Murata N, Date S, Inoue M. 2007. Cell death in seedlings of the interspecific hybrid of Nicotiana gossei and N. tabacum; possible role of knob-like bodies formed on tonoplast in vacuolar-collapse-mediated cell death. Plant Cell Reports 26: 407-419.

Müller J, Beck M, Mettbach U, Komis G, Hause G, Menzel D, Samaj J. 2010. Arabidopsis MPK6 is involved in cell division plane control during early root development, and localizes to the pre-prophase band, phragmoplast, trans-Golgi network and plasma membrane. Plant Journal 61: 234-248.

Mur LAJ, Aubry S, Mondhe M, Kingston-Smith A, Gallagher J, TimmsTaravella E, James C, Papp I, Hörtensteiner S, Thomas H et al. 2010. Accumulation of chlorophyll catabolites photosensitizes the hypersensitive response elicited by Pseudomonas syringae in Arabidopsis. New Phytologist 188: 161-174.

Ng CKY, Carr K, McAinsh MR, Powell B, Hetherington AM. 2001. Drought-induced guard cell signal transduction involves sphingosine-1phosphate. Nature 410: 596-599.

Pandey S, Assmann SM. 2004. The Arabidopsis putative G proteincoupled receptor GCR1 interacts with the G protein $\alpha$ subunit GPA1 and regulates abscisic acid signaling. Plant Cell 16: 1616-1632.

Pata MO, Hannun YA, Ng CKY. 2009. Plant sphingolipids: decoding the enigma of the sphinx. New Phytologist 185: 611-630.

Peer M, Stegmann M, Mueller MJ, Waller F. 2010. Pseudomonas syringae infection triggers de novo synthesis of phytosphingosine from sphinganine in Arabidopsis thaliana. Federation of Biochemical Societies Letters 584: 4053-4056.

Pinelli E, Poux N, Garren L, Pipy B, Castegnaro M, Miller DJ, PfohlLeszkowicz A. 1999. Activation of mitogen-activated protein kinase by fumonisin $\mathrm{B}(1)$ stimulates $\mathrm{CPLA}(2)$ phosphorylation, the arachidonic acid cascade and cAMP production. Carcinogenesis 20: 1683-1688.

Pitzschke A, Djamei A, Bitton F, Heribert H. 2009. A major role of the MEKK1-MKK1/2-MPK4 pathway in ROS signaling. Molecular Plant 2: $120-137$.

Popescu SC, Popescu GV, Bachan S, Zhang Z, Gerstein M, Snyder M, Dinesh-Kumar S. 2009. MAPK target networks in Arabidopsis thaliana revealed using functional protein microarrays. Genes and Development 23: 80-92.

Ren D, Liu Y, Yang KY, Han L, Mao G, Glazebrook J, Zhang S. 2008. A fungal-responsive MAPK cascade regulates phytoalexin biosynthesis in Arabidopsis. Proceedings of the National Academy of Sciences, USA 105: 5638-5643.

Ren D, Yang K, Li G, Liu Y, Zhang S. 2006. Activation of Ntf4, a tobacco mitogen-activated protein kinase, during plant defense response and its involvement in hypersensitive response-like cell death. Plant Physiology 141: 1482-1493.

Ren D, Yang H, Zhang S. 2002. Cell death mediated by MAPK is associated with hydrogen peroxide production in Arabidopsis. Journal of Biological Chemistry 277: 559-565.

Schroeder JI, Kwak JM, Allen GJ. 2001. Guard cell abscisic acid signalling and engineering drought hardiness in plants. Nature 410: 327-330.

Shi L, Bielawski J, Mu J, Dong H, Teng C, Zhang J, Yang X, Tomishige N, Hanada K, Hannun YA et al. 2007. Involvement of sphingoid bases in mediating reactive oxygen intermediate production and programmed cell death in Arabidopsis. Cell Research 17: 1030-1040.
Stone JM, Heard JE, Asai T, Ausubel FM. 2000. Simulation of fungalmediated cell death by fumonisin B1 and selection of fumonisin B1resistant (fbr) Arabidopsis mutants. Plant Cell 12: 1811-1822.

Takahashi Y, Berberich T, Kanzaki H, Matsumura H, Saitoh H, Kusano T, Terauchi R. 2009. Serine palmitoyltransferase, the first step enzyme in sphingolipid biosynthesis, is involved in nonhost resistance. Molecular Plant-Microbe Interactions 22: 31-38.

Tao Y, Xie Z, Chen W, Glazebrook J, Chang HS, Han B, Zhu T, Zou G, Katagiri F. 2003. Quantitative nature of Arabidopsis responses during compatible and incompatible interactions with the bacterial pathogen Pseudomonas syringae. Plant Cell 15: 317-330.

Townley HE, McDonald K, Jenkins GI, Knight MR, Leaver CJ. 2005. Ceramides induce programmed cell death in Arabidopsis cells in a calcium-dependent manner. Biological Chemistry 386: 161-166.

Tsegaye Y, Richardson CG, Bravo JE, Mulcahy BJ, Lynch DV, Markham JE, Jaworski JG, Chen M, Cahoon EB, Dunn TM. 2007. Arabidopsis mutants lacking long chain base phosphate lyase are fumonisin-sensitive and accumulate trihydroxy-18:1 long chain base phosphate. Journal of Biological Chemistry 282: 28195-28206.

Turner S, Gallois P, Brown D. 2007. Tracheary element differentiation. Annual Review of Plant Biology 58: 407-433.

Ulm R, Ichimura K, Mizoguchi T, Peck SC, Zhu T, Wang X, Shinozaki K, Paszkowski J. 2002. Distinct regulation of salinity and genotoxic stress responses by Arabidopsis MAP kinase phosphatase 1. European Molecular Biology Organization Journal 21: 6483-6493.

Ulm R, Revenkova E, di Sansebastiano GP, Bechtold N, Paszkowski J. 2001. Mitogen-activated protein kinase phosphatase is required for genotoxic stress relief in Arabidopsis. Genes and Development 15: 699-709.

Walley JW, Coughlan S, Hudson ME, Covington MF, Kaspi R, Banu G, Harmer SL, Dehesh K. 2007. Mechanical stress induces biotic and abiotic stress responses via a novel cis-element. PLoS Genetics 3: $1800-1812$

Wang E, Norred WP, Bacon CW, Riley RT, Merrill AH Jr. 1991. Inhibition of sphingolipid biosynthesis by fumonisins. Journal of Biological Chemistry 266: 14486-14490.

Wang H, Ngwenyama N, Liu Y, Walker JC, Zhang S. 2007. Stomatal development and patterning are regulated by environmentally responsive mitogen-activated protein kinases in Arabidopsis. Plant Cell 19: 63-73.

Wang W, Yang X, Tangchaiburana S, Ndeh R, Markham JE, Tsegaye Y, Dunn TM, Wang GL, Bellizzi M, Parsons JF et al. 2008. An inositolphosphorylceramide synthase is involved in regulation of plant programmed cell death associated with defense in Arabidopsis. Plant Cell 20: 3163-3179.

Wang X. 2004. Lipid signaling. Current Opinion in Plant Biology 7: 329-336.

Wattenberg EV, Badria FA, Shier WT. 1996. Activation of mitogen-activated protein kinase by the carcinogenic mycotoxin fumonisin B1. Biochemical and Biophysical Research Communications 227: 622-627.

Worrall D, Liang YK, Alvarez S, Holroyd GH, Spiegel S, Panagopulos M, Gray JE, Hetherington AM. 2008. Involvement of sphingosine kinase in plant cell signaling. Plant Journal 56: 64-72.

Yang CH, Gavilanes-Ruiz M, Okinaka Y, Vedel R, Berthuy I, Boccara M, Chen JW, Perna NT, Keen NT. 2002. hrp genes of Erwinia chrysanthemi 3937 are important virulence factors. Molecular Plant-Microbe Interactions 15: 472-480.

Yao N, Greenberg JT. 2006. Arabidopsis ACCELERATED CELL DEATH2 modulates programmed cell death. Plant Cell 18: 397-411.

Zhang S, Du H, Klessig DF. 1998. Activation of the tobacco SIP kinase by both a cell wall-derived carbohydrate elicitor and purified proteinaceous elicitins from Phytophthora spp. Plant Cell 10: 435-450. 
New

Zhang S, Liu Y, Klessig DF. 2000. Multiple levels of tobacco WIPK activation during the induction of cell death by fungal elicitins. Plant Journal 23: 339-347.

Zhang Y, Zhu H, Zhang Q, Li M, Yan M, Wang R, Wang L, Welti R, Zhang W, Wang K. 2009. Phospholipase D $\alpha 1$ and phosphatidic acid regulate NADPH oxidase activity and production of reactive oxygen species in ABA-mediated stomatal closure in Arabidopsis. Plant Cell 21: $2357-2377$.

\section{Supporting Information}

Additional supporting information may be found in the online version of this article.

Fig. S1 Modification of long chain bases during de novo synthesis of sphingolipids in plants.

Fig. S2 Programmed cell death promoted by fumonisin B1 (FB1) is already observed at $36 \mathrm{~h}$.

Fig. S3 Accumulation kinetics of the less abundant free long chain bases (LCBs) and phosphorylated LCBs (LCBs-
P) in wild-type (WT) and $l c b 2 a-1$ seedlings exposed to fumonisin B1 (FB1).

Table S1 Accumulation kinetics of free long chain bases and phosphorylated long chain bases in wild-type (WT) and $l c b 2 a-1$ mutant seedlings treated or not with fumonisin $\mathrm{B} 1$ (FB1)

Methods S1 Plant material and growth conditions, isolation of T-DNA insertion lines and construction of mutant lines, detection of nuclear DNA fragmentation, ultrastructural analysis, protein extraction and kinase assays, and immunoblots of MPK6 and MPK3.

Please note: Wiley-Blackwell are not responsible for the content or functionality of any supporting information supplied by the authors. Any queries (other than missing material) should be directed to the New Phytologist Central Office.

\section{About New Phytologist}

- New Phytologist is owned by a non-profit-making charitable trust dedicated to the promotion of plant science, facilitating projects from symposia to open access for our Tansley reviews. Complete information is available at www.newphytologist.org.

- Regular papers, Letters, Research reviews, Rapid reports and both Modelling/Theory and Methods papers are encouraged We are committed to rapid processing, from online submission through to publication 'as-ready' via Early View - our average submission to decision time is just 29 days. Online-only colour is free, and essential print colour costs will be met if necessary. We also provide 25 offprints as well as a PDF for each article.

- For online summaries and ToC alerts, go to the website and click on 'Journal online'. You can take out a personal subscription to the journal for a fraction of the institutional price. Rates start at $£ 149$ in Europe/\$276 in the USA \& Canada for the online edition (click on 'Subscribe' at the website).

- If you have any questions, do get in touch with Central Office (np-centraloffice@lancaster.ac.uk; tel +44 1524 594691) or, for a local contact in North America, the US Office (newphytol@ornl.gov; tel +1 8655765261 ). 